Service social

\title{
La prévention des problèmes sociaux : réalité québécoise
}

\section{Claire Chamberland, Nicole Dallaire, Sylvie Cameron, Lucie Fréchette, Jacques Hébert et Jocelyn Lindsay}

Volume 42, numéro 3, 1993

Perspectives théoriques

URI : https://id.erudit.org/iderudit/706631ar

DOI : https://doi.org/10.7202/706631ar

Aller au sommaire du numéro

Éditeur(s)

École de service social de l'Université Laval

ISSN

1708-1734 (numérique)

Découvrir la revue

Citer cet article

Chamberland, C., Dallaire, N., Cameron, S., Fréchette, L., Hébert, J. \& Lindsay, J. (1993). La prévention des problèmes sociaux : réalité québécoise. Service social, 42(3), 55-81. https://doi.org/10.7202/706631ar

\section{Résumé de l'article}

Les modèles de prévention actuels et leurs stratégies d'action contribuent-ils à une diminution importante et durable des problèmes sociaux? La réponse à cette question suppose qu'on examine la représentation des problèmes sociaux, les cibles et les contextes de prévention, la dimension de précocité de l'action ainsi que les stratégies privilégiées. Les développements théoriques actuels permettent d'acquérir une vision liant la résolution de problèmes individuels et celle de problèmes sociaux. Les auteurs dégagent enfin une première esquisse de la prévention sociale. La conjoncture actuelle nous laisse croire à une évolution intéressante de ce secteur dans la prochaine décennie. 


\section{La prévention des problèmes sociaux: réalité québécoise}

Claire CHAMBERLAND

Professeure

École de service social, Université de Montréal

Nicole DALLAIRE

Étudiante au doctorat en sciences humaines et appliquées

Université de Montréal

Sylvie CAMERON

Professionnelle de recherche École de service social, Université de Montréal

Lucie FRÉCHETTE

Professeure

Département des sciences humaines

Université du Québec à Hull

Jacques HÉBERT

Professeur

Département de travail social Université du Québec à Montréal

Jocelyn LINDSAY

Professeur

École de service social, Université Laval

Les problèmes sociaux apparaissant dans une collectivité expriment bien plus qu'un désarroi personnel: ils reflètent avant tout l'existence d'un véritable malaise de civilisation. Ils sont en quelque sorte des épiphénomènes d'une réalité plus globale. Dans le présent article, nous aborderons 
la question de la prévention des principales problématiques auxquelles sont confrontés les services sociaux en interrogeant les modèles de prévention actuels, de même que les stratégies d'action les plus prometteuses par rapport à ce qui nous apparaît être un objectif incontournable, même s'il est ambitieux: la diminution importante et durable des problèmes sociaux.

Pour ce faire, nous préciserons les principaux éléments à la base d'une définition acceptable de la prévention en identifiant les questions cruciales auxquelles on doit répondre pour planifier les actions préventives. Les principaux modèles qui ont déterminé le développement des pratiques préventives seront examinés et évalués en fonction de leur habileté à générer des objectifs et des stratégies susceptibles de réduire les risques et d'améliorer de manière significative le sort des individus et des communautés. Par la suite, nous tracerons les principales influences qui ont favorisé un rapprochement conceptuel entre problèmes individuels et problèmes sociaux. Enfin, nous proposerons une vision où la résolution des problèmes individuels est étroitement liée à la résolution des problèmes sociaux. En guise de conclusion, une définition préliminaire de la prévention sociale sera esquissée.

\section{ÉLÉMENTS PRÉALABLES à UNE DÉFINITION DE LA PRÉVENTION}

Le champ de la prévention est essentiellement pratique et se distingue des autres types d'intervention sous deux aspects: il comporte un élément de précocité, eu égard à la manière dont le problème est défini (et implique donc un acte conscient d'anticipation de la part de l'intervenant) et cible des agrégats (population ou communauté) plutôt que des individus (Bloom, 1981; Low, 1979; Pransky, 1991). Les approches préventives ne sont associées ni à une idéologie politique spécifique ni, encore moins, à un modèle théorique particulier (Lafortune et Kieley, 1989). Comme nous le constaterons plus loin, les différentes stratégies reposent sur des raisonnements très variés et font appel à des approches consensuelles, alors que d'autres sont de nature plus conflictuelle (Moreau, 1987). En effet, les modèles explicatifs et les schémas d'interprétation des chercheurs et des intervenants conditionnent largement leurs choix stratégiques.

Au Québec, l'intervention psychosociale semble enfin être marquée par ce que Albee (1980) définissait comme une quatrième révolution paradigmatique dans l'intervention en santé mentale, soit la montée croissante d'initiatives en matière de prévention. En effet, un nombre de plus en plus grand de personnes remettent en question le fait que la presque totalité du budget dans les services sociaux soit dirigée vers des clientè- 
les aux prises avec des problèmes aigus. Un virage important est souhaité et parfaitement bien exprimé dans les intentions du rapport du Groupe de travail sur les jeunes (Gouvernement du Québec, 1991):

Notre Groupe a plutôt opté pour une approche qui mise sur une intervention très précoce dans la vie des enfants et qui investit dans la mise en place de contextes capables de les orienter le plus tôt possible sur des voies où l'affection, la réussite et l'acquisition $d^{\prime}$ un sentiment d'identité et de confiance personnelle deviennent les meilleurs instruments de protection. Il faut investir dans l'amélioration des milieux de vie des enfants (la famille, les garderies, l'école, le voisinage) si on veut qu'ils y développent les compétences qui les éloigneront des services de santé et de réadaptation (p. 162).

La réponse à cinq grandes questions oriente de manière significative le travail des chercheurs, gestionnaires et praticiens en matière de prévention: quels problèmes voulons-nous prévenir (le quoi), quelles seront les clientèles ou populations (vers qui), dans quels contextes ou milieux interviendrons-nous (où), à quel moment dans l'évolution du problème (quand) et, enfin, quelles seront les stratégies privilégiées (comment)?

\section{LA REPRÉSENTATION DES PROBLÈMES SOCIAUX}

Une vision claire d'un plan d'action en matière de prévention nécessite que l'on délimite clairement la nature des problèmes (le quoi) que l'on cherche à prévenir. Ce consensus peut s'établir grâce à une définition commune par les chercheurs et les intervenants des indicateurs associés à un problème, lesquels permettent que tous et chacun acceptent de jeter un regard similaire sur la réalité des difficultés vécues par les jeunes. De plus, pour qu'un consensus émerge autour de l'urgence de prévenir, une certaine congruence dans les perceptions des gestionnaires et politiciens constitue une condition facilitante supplémentaire. Ainsi s'élabore un processus collectif de définition par lequel un ensemble de faits est reconnu comme un problème social par différents acteurs, alors que $d^{\prime}$ autres conditions, tout aussi nuisibles, ne le sont pas (Mayer et Laforest, 1990). Des facteurs historiques et politiques constituent de puissants déterminants de ce même processus. À preuve, depuis plus d'une décennie la société québécoise a progressivement "découvert » les phénomènes de négligence et $d^{\prime}$ abus physique et sexuel faits aux enfants (Massé, 1992). Elle est sur le point de découvrir que des mots ou des gestes peuvent parfois faire aussi mal qu'une agression physique. Une nouvelle étiquette est en voie d'apparaître, soit l'abus émotionnel ou encore la violence psychologique (ou mauvais traitement psychologique), phénomène pour lequel 
la collectivité n'a pas encore élaboré de vocabulaire bien précis (Fortin, 1992). En somme, ériger des difficultés d'adaptation en problèmes sociaux susceptibles de mobiliser différentes catégories d'acteurs implique que ces mêmes acteurs établissent un certain consensus quant à la réalité de ces problèmes et de leur définition et à ce qui est socialement tolérable (Schneider, 1985).

Récemment, le Groupe de travail pour les jeunes fournissait un portrait global des types et de l'ampleur des principales difficultés majeures des jeunes dans un rapport intitulé Un Québec fou de ses enfants (Gouvernement du Québec, 1991). Trois grandes catégories de problèmes ont dès lors été mises au jour: les troubles de victimisation, d'externalisation ainsi que de décrochage. Dans le premier cas, on fait référence principalement aux mauvais traitements infligés aux enfants. Dans le second cas, les problèmes regroupés sous cette rubrique concernent les phénomènes de délinquance, de violence ou de troubles de comportements, ces trois formes de mésadaptations étant généralement très associées entre elles. Outre les problèmes de violence ou de délinquance, deux autres types de mésadaptations sont considérés comme troubles d'externalisation: la toxicomanie ainsi que la grossesse à l'adolescence. Dans le troisième cas, le phénomène de décrochage est envisagé de quatre manières différentes: décrochage de l'école (abandon scolaire), de la famille (les enfants placés), de la communauté (l'itinérance) ou encore de la vie (le suicide). Outre par ces trois grands regroupements de problèmes, le rapport Un Québec fou de ses enfants s'est également distingué en conférant un statut particulier à un problème social qui afflige une proportion importante de Québécois et de Québécoises. Il s'agit de la pauvreté économique qui accentue dangereusement la précarité des milieux de vie.

En plus de la publication de ce rapport, un second événement témoigne d'une volonté politique, véritable révélateur $d^{\prime} u n$ courant social de plus en plus manifeste dans le réseau des services sociaux et de santé au Québec: la parution de la Politique de la santé et du bien-être en 1992. Parmi les 19 problèmes qui affectent le plus les Québécois, six concernent directement les troubles $d^{\prime}$ adaptation sociale, deux relèvent plus directement d'une problématique en santé mentale et, enfin, deux autres objectifs s'articulent autour de l'intégration sociale des personnes âgées et des handicapés. Sur les objectifs considérés comme prioritaires, dix concernent directement ou indirectement les problématiques sociales, alors que les neuf autres sont davantage formulés dans une perspective de santé physique ou de santé publique. Enfin, six objectifs sur dix-neuf font référence aux réalités vécues par les jeunes et coïncident globalement avec les représentations exprimées dans le rapport Un Québec fou de ses 
enfants. Dans ce dernier rapport, la problématique de la pauvreté $n^{\prime}$ est pas explicitement abordée comme problème à prévenir, mais est considérée dans le chapitre des stratégies. Selon les auteurs, I'amélioration des conditions de vie représente un des moyens pour réduire les différents problèmes de santé ou d'adaptation sociale. Les deux rapports ont néanmoins le mérite de permettre que, collectivement, le Québec reconnaisse et conçoive différentes facettes des problématiques qui affectent la vie de la population québécoise, le nombre de personnes touchées, les effets néfastes que ces problématiques engendrent et l'existence de solutions efficaces (Gouvernement du Québec, 1991, 1992).

En somme, l'identification du quoi prévenir est indissociable des acteurs participant à sa définition et de la dynamique sociale dans laquelle ce processus de définition s'inscrit (enjeux politiques et institutionnels, résistance au changement, etc.). Actuellement, la publication des deux rapports mentionnés précédemment permet de penser qu'il existe une entente conjoncturelle sur un certain nombre de priorités en matière de prévention, et tout au moins sur l'urgence de réduire à moyen terme les coûts des services sociaux de santé dans un contexte économique où les goussets de nos gouvernements sont désespérément vides. Mais de la coupe aux lèvres, il y a une distance pas toujours aisée à franchir.

\section{LES CIBLES ET LES CONTEXTES EN PRÉVENTION}

La réponse aux questions suivantes est également cruciale dans la planification de programmes préventifs. Quels jeunes et quelles familles seront prioritairement ciblés? Dans quels milieux ou dans quelles communautés injecterons-nous les fonds nécessaires pour implanter les programmes? Conserverons-nous une approche universelle des soins de santé et de services sociaux à l'ensemble du Québec, une approche plus orientée vers des clientèles à risque ou encore viserons-nous des communautés aux prises avec de grandes difficultés mais dont les services seront accessibles dans I'ensemble du territoire où elles vivent et survivent? Malgré une préférence marquée pour une approche par territoire ainsi que la pertinence du principe de l'accessibilité pour tous clairement affirmée dans le rapport Un Québec fou de ses enfants, une tendance assez claire se dessine en faveur d'une approche axée vers les clientèles à risque, déjà bien esquissée dans le rapport Brunet (1987). Et pourtant d'aucuns soulignent, à juste titre, les imperfections et les effets pervers d'une telle orientation, notamment l'existence de jeunes ou de familles non repérés par l'appareil diagnostique, mais qui vivront des difficultés (les faux négatifs, selon Chamberlin, 1984), ou encore l'impact stigmatisant résultant du 
processus de l'étiquetage "être à risque (Goffman, 1975; Rosenthal et Jacobson, 1968).

\section{L'IMPORTANCE DE LA PRÉCOCITÉ DE L'ACTION}

La quatrième question renvoie à la chronologie des événements qui caractérise l'évolution d'un problème et du moment dans lequel survient l'intervention compte tenu du degré d'aggravation de la situation problème. Plus concrètement, on fait référence ici aux notions de promotion et de prévention primaire, secondaire et tertiaire, la différence entre ces niveaux se situant au moment où I'on intervient pour résoudre un problème, étant donné sa progression dans le temps (voir à ce sujet Bloom, 1981 ; Low, 1992).

Les objectifs de la promotion s'insèrent d'emblée dans le cadre $d^{\prime}$ une dynamique positive. On parle davantage de déterminants et de conditions favorables au développement plutôt que de problèmes à éviter ou à résoudre (Gouvernement du Québec, 1990). La prévention primaire $s^{\prime}$ attache plus particulièrement à court-circuiter l'apparition de problèmes qui pourraient survenir chez une personne ou une communauté en créant les conditions favorisant la santé globale pour la population en général et même pour des groupes présentant des risques susceptibles d'entraîner des problèmes. Il s'agit $d^{\prime}$ intervenir le plus possible de façon proactive par l'élimination des facteurs prédisposants (soit la neutralisation d'influences pathogènes) ou par l'addition de facteurs positifs de nature à améliorer le répertoire de ressources individuelles (compétences, résistance au stress, appropriation) et environnementales (climat familial, logement, emploi, revenu et conditions de travail, soutien social). En promotion ou en prévention primaire, l'action se déroule généralement dans ce que Bower (1990) appelle les systèmes sociaux clés d'intégration ou les institutions de base, tels la famille, l'école, les milieux de garde, le réseau social et la communauté, de même que les services de première ligne ayant plutôt un mandat de prévention (par ex. les centres locaux de services communautaires au Québec).

La prévention secondaire intervient une fois que certains symptômes apparaissent ou que certains signes avant-coureurs (l'analogie en médecine serait le prodrome) signalent la présence d'une dysfonction en progression. L'objectif vise ainsi avant tout à éviter l'aggravation des problèmes en écourtant la durée moyenne des cas problématiques déjà présents; on intervient dès lors sur les facteurs précurseurs. Les stratégies déployées consistent alors à réduire les crises à court terme, à acquérir des compétences pour mieux aider l'individu à répondre aux situations 
problématiques, ou à modifier certaines conditions de la situation problématique.

La prévention tertiaire ne serait pas réellement de la prévention, puisqu'elle vise essentiellement la réhabilitation ou l'arrêt de comportements déviants (alcoolisme, toxicomanie, violence familiale, etc.) et qu'elle cible les personnes ayant d'assez graves troubles de comportements ou de santé. L'intervention est davantage de nature clinique dans la mesure où l'on agit sur la résorption du symptôme par le moyen de thérapies ou en développant des habiletés pour favoriser le retour des personnes à un fonctionnement considéré comme «normal ». À ce niveau, la prévention fait plutôt appel au traitement curatif, la dimension plus spécifiquement préventive résidant dans l'évitement éventuel d'une récidive.

En prévention secondaire et tertiaire, l'intervention implique bien souvent des institutions souffrantes ou en difficulté (les SED selon Bower, 1990), telles que les cliniques pédopsychiatriques, les écoles spécialisées, les institutions ayant un mandat de protection de la jeunesse... Dans d'autres cas, on doit avoir recours à une troisième catégorie d'institutions dont l'objectif est de contenir et de minimiser les risques que des personnes peuvent présenter à la fois pour elles-mêmes et pour la société. Les établissements correctionnels et psychiatriques (ECP selon Bower, 1990) constituent des milieux où sont fournis des services spécialisés auxquels on a recours lorsque les services dits de première et de deuxième ligne ont échoué à faire cesser la progression du problème. Pour certains, l'intervention effectuée dans ces milieux a une fonction de réhabilitation (donc de nature tertiaire).

En somme, la promotion et la prévention primaire sont les seuls types d'intervention permettant d'agir sur une catégorie de facteurs qui surviennent avant l'apparition d'un problème et dont l'objectif vise explicitement à empêcher l'apparition de nouveaux cas (taux de fréquence). Pour plusieurs auteurs, on devrait réserver l'appellation prévention à des actions de type primaire ou promotionnel (Albee, 1980; Lafortune et Kiely, 1989).

\section{DES STRATÉGIES LIÉES À LA COMPRÉHENSION DES PROBLÈMES SOCIAUX}

Une dernière question, et non la moindre, complète la liste des interrogations; quelles seront les stratégies pertinentes à privilégier dans le réseau des services sociaux afin de pouvoir espérer voir chuter les taux de problèmes chez les jeunes? La compréhension que nous avons de 
l'étiologie d'un problème social oriente de manière importante les objectifs ainsi que la nature des moyens mis en branle pour contrer ce problème. L'adhésion à un modèle théorique suppose une centration sur certains types de facteurs jugés prioritaires en occultant nécessairement d'autres causes potentiellement déterminantes (Catalano, 1979). Trois grandes approches ont influencé le champ de I'action préventive: le modèle biomédical, le modèle de la santé communautaire et le modèle écologique (Gouvernement du Québec, 1985a).

Le modèle biomédical se centre sur l'étude des caractéristiques personnelles et familiales, qui représentent les principaux déterminants du problème social. L'intervention préventive est souvent de niveau tertiaire et parfois secondaire dans la mesure où son action est plus dirigée vers l'éradication des problèmes et des risques déjà présents dans l'environnement de l'hôte (Chamberland, à paraître). Ainsi les cibles concernent surtout des populations dites à risque et même des clientèles présentant des signes avant-coureurs et chez qui même les problèmes sont déjà bien manifestes. Les stratégies privilégiées par ce modèle visent principalement à réduire et à corriger les déficits du jeune, des parents ou de la famille ou à neutraliser l'impact d'un passé douloureux du parent sur le développement de l'enfant. Les interventions sont d'inspiration plus clinique.

Le modèle de santé communautaire fait la distinction entre les facteurs prédisposants (caractéristiques de l'individu et des milieux de vie) et les facteurs précipitants (événements stressants). II soulève également le rôle prépondérant de facteurs de protection et d'opportunités chez I'individu (compétences) et ses contextes de vie (soutien social) qui peuvent amoindrir $d^{\prime}$ une certaine façon la gravité du problème vécu par les personnes ou la famille. À cet égard, ce modèle favorise une prévention de niveau secondaire et primaire dans la mesure où il permet $d^{\prime}$ anticiper l'apparition éventuelle de situations à risque ou de développer des facteurs de protection afin de modérer les effets directs et indirects du stress sur les individus. Les actions sont toutefois généralement limitées aux milieux de vie des personnes (microsystèmes et mésosystème) et ciblent autant la population dite en santé que celle exposée à des facteurs de risque. En somme, ce modèle permet d'intervenir plus tôt dans la genèse des problèmes, de même qu'il considère les caractéristiques négatives et positives des personnes et des contextes proches qui interfèrent ou facilitent l'adaptation des personnes dans leur milieu. Les stratégies les plus couramment associées à ce modèle sont souvent de nature plus éducative et axées vers le développement des compétences ou la promotion du soutien social.

Enfin le modèle écologique, avec son analyse plus holistique, a élargi le spectre des facteurs de risque ou d'opportunités en mentionnant 
aussi le rôle important des environnements éloignés dans l'étiologie des problèmes sociaux. Ainsi, l'organisation des sociétés et leur volonté de participer ou non au bien-être des populations (ex.: les décisions d'ordre politique et économique dans les institutions sociales tant privées que publiques) ainsi que les valeurs sociales véhiculées participent également à l'apparition des problèmes sociaux. De plus, le modèle écologique a contribué à faire émerger une nouvelle compréhension des problèmes sociaux par son insistance à examiner les interrelations complexes qui relient l'individu à son environnement en accordant à celui-ci un statut particulier. Ce modèle tient compte plus particulièrement de la synergie qui existe dans une configuration de facteurs de risque (loi de la multiplication des effets) ainsi que du poids relatif et changeant d'un déterminant compte tenu des facteurs qui sont simultanément présents (loi de la relativité) (Bronfenbrenner, à paraître; Chamberland, à paraître). Les actions préventives peuvent avoir pour cibles les individus, leurs milieux de vie ainsi que les environnements éloignés, qu'ils soient ou non exposés à des facteurs de risque. Par conséquent, la prévention s'appuyant sur ce modèle se situe davantage à un niveau primaire et même promotionnel. Elle peut viser le développement des compétences, tout en favorisant également l'accroissement du soutien social et la mise en place d'une mobilisation de la population pour faire changer l'orientation d'une politique sociale. En bref, les actions préventives qu'elle promeut sont dirigées vers des systèmes dynamiques, multiples et interdépendants (Laurendeau et al., 1990; Bouchard, 1987; Kelly, 1968; Vincent et Trickett, 1983). Les stratégies axées vers le développement de compétences, I'amélioration des processus de communication parent-enfant côtoient le développement communautaire, le marketing social ou I'action politique.

L'évolution des différents modèles théoriques a ainsi permis de comprendre que les problèmes psychosociaux (ex. : la négligence envers les enfants), qui sont souvent attribués aux personnes ou aux familles, peuvent être des "symptômes", des manifestations ou des effets d'une configuration de facteurs de risque environnementaux (Pransky, 1991).

On peut douter de l'efficacité de l'arsenal classique des pratiques préventives dont les seuls objectifs sont orientés vers le développement des compétences, la résistance au stress ou le développement du soutien social, en évacuant de leur perspective la contribution des facteurs macrosociaux. En d'autres termes, les programmes de prévention dont le cadre $d^{\prime}$ analyse et les choix stratégiques s'inspirent des modèles biomédical et de santé communautaire limiteraient sérieusement la portée véritable des pratiques préventives. Nous retracerons brièvement les principaux courants d'influence qui ont jeté progressivement les bases d'un rapprochement entre l'analyse des problèmes individuels et les problèmes sociaux. 
En présentant ainsi trois grandes approches qui ont influencé le champ de l'action préventive, nous sommes conscients des deux limites suivantes: d'une part, les modalités concrètes d'intervention sont esquissées, mais non élaborées; d'autre part, il reste à préciser une jonction aux grandes stratégies de changement dans les organisations humaines, telles que formulées par exemple par Chin et Benne (1985). Ceux-ci définissent des stratégies empiriques-rationnelles (changement provoqué par la connaissance et la logique d'un gain apporté par un changement), les stratégies normatives-éducatives (changement outrepassant l'aspect rationnel et ciblant les attitudes et les valeurs pour modifier les normes socioculturelles) et les stratégies de pouvoir ou d'ordre politique (utilisation des différentes formes de pouvoir et des rapports de force).

\section{FORMULATION DES PROBLÈMES INDIVIDUELS EN PROBLÈMES SOCIAUX: LA POINTE OU L'ICEBERG?}

\section{La contribution d'Albee et de Pransky en prévention}

Albee fut l'un des premiers spécialistes en prévention à présenter les inégalités sociales comme facteur contribuant à la détérioration de la santé mentale des gens. Il a également soulevé la nécessité de mener des actions politiques afin de réduire ces inégalités et d'étendre la portée des pratiques préventives inspirées du modèle biomédical. Des actions portées au niveau du système socio-économique seraient l'approche la plus efficace pour prévenir les psychopathologies (Albee, 1986). Albee dénonce la tendance à isoler des problèmes sociaux les différents stresseurs que vivent les individus (ex.: toutes les formes de violence vécues par les enfants, les femmes, les personnes âgées). Cette séparation tente de masquer les caractéristiques communes à toutes les personnes victimes de sexisme, de racisme, de pauvreté, d'absence de pouvoir - (powerlessness) - dont les conditions économiques et politiques sont à la base de ces phénomènes d'exclusion sociale. Il a ainsi participé à l'élargissement du champ de l'analyse et de l'action préventive, dans le secteur de la santé mentale, en tenant compte des facteurs sociaux, politiques et économiques contribuant au développement des problèmes de santé mentale.

L'une des principales contributions d'Albee fut l'élaboration d'une équation identifiant la présence $d^{\prime}$ 'une configuration de facteurs de risque et de facteurs d'opportunités dans la fréquence des désordres psychosociaux.

\begin{tabular}{|c|c|c|c|c|c|c|}
\hline \multirow{2}{*}{ fréquence } & & stress & + & facteurs organiques & + & exploitation \\
\hline & & $\begin{array}{c}\text { adaptation } \\
\text { (coping) }\end{array}$ & + & estime de soi & + & soutien social \\
\hline
\end{tabular}


Selon Albee, la réduction de la fréquence de désordres psychosociaux doit passer par une intervention préventive visant à modifier cette configuration, soit en abaissant la présence des facteurs de risque, soit en augmentant la présence des facteurs d'opportunités.

Pransky (1991) a clarifié l'équation d'Albee en propositions opérationnelles plus concrètes. En ce sens, il mentionne que I'addition de nombreux facteurs de risque augmente la fréquence de problèmes. Ces facteurs se situent dans les environnements éloignés de l'individu, dans ses milieux de vie significatifs ou chez la personne elle-même. Ainsi les risques sont simultanément présents dans une multitude de systèmes: les attentes culturelles (violence dans les médias, les enfants et les femmes considérés comme objets de propriété), les stresseurs socio-environnementaux (chômage, stress résultant des lois sauvages de l'économie), l'impossibilité d'atteindre des buts socialement acceptables en raison d'un manque d'opportunités (argent, prestige, statut, estime des autres) et, enfin, les dysfonctionnements familiaux (tensions et conflits) ou organiques (handicaps menant à la discrimination...). Améliorer les capacités de résistance des individus implique aussi qu'on dirige nos interventions vers le développement $d^{\prime}$ une perception de soi positive, la promotion d'habiletés sociales ou d'un certain processus de conscientisation (awareness). Les lieux d'intervention sont la famille, l'école, les pairs, le milieu de travail, la communauté et les politiques sociales. L'équation suivante illustre la perspective de Pransky (1991).

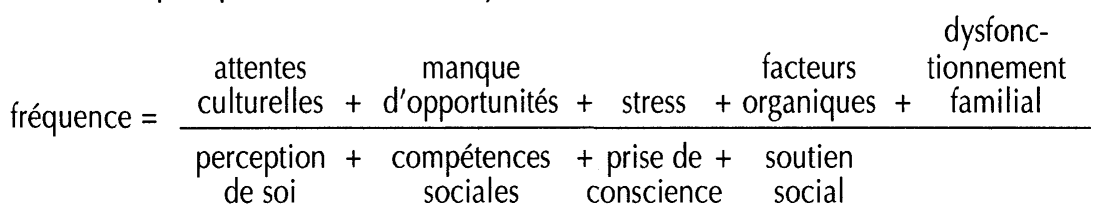

De plus, les facteurs de risque et de protection sont communs à une variété de problématiques (Gouvernement du Québec, 1991, 1992; Pransky, 1991). C'est ce qui permet de conclure que la promotion et la prévention primaire ont un spectre plus large d'impact et que par conséquent leur perspective semble plus générale. En effet, un même facteur prédisposant peut provoquer plusieurs effets différents. Ainsi agir sur l'amélioration des compétences parentales ou des conditions de vie peut empêcher l'émergence de la violence, de la négligence ou de la délinquance. D'où la puissance d'une approche proactive et orientée vers des facteurs de risque et de protection de type générique, ainsi que le soulignent Albee (1982, 1983) et Pransky (1991). 


\section{La contribution de la psychologie communautaire}

Les travaux d'Albee et de Pransky sur les facteurs favorisant la santé mentale et le bien-être sont étroitement reliés au champ de la psychologie communautaire. Depuis dix ans, I'analyse de l'influence des facteurs sociaux et environnementaux sur le comportement est devenue centrale en psychologie communautaire, de même que la nécessité de conceptualiser l'intervention de manière plus proactive (Klein, 1987).

Le modèle en santé communautaire avait, rappelons-le, déplacé notre attention vers l'étude des causes générales et non spécifiques pour expliquer l'émergence des problèmes émotionnels; $c^{\prime}$ est ce qui lui a permis d'établir une distinction entre les facteurs prédisposants et les facteurs précipitants (Bloom, 1979 et 1981) et de reconnaître graduellement l'existence de certains dénominateurs communs entre problèmes psychosociaux et problèmes sociaux. Par la suite, l'approche écologique a fourni les bases conceptuelles pour compléter cette triple mutation dans la formulation de ce qu'est un problème. De problème individuel, il est devenu psychosocial, dans la mesure où l'on reconnaissait la présence de déterminants à la fois personnels et environnementaux. Puis de psycho-social il a de plus en plus été qualifié de problème social, puisque le dysfonctionnement individuel est perçu davantage comme révélateur de la qualité d'un environnement (Chamberland et Beaudry, 1989). Par analogie, le taux de mauvais traitement dans une communauté serait un indice de pollution sociale. Ou encore le drame de la drogue ou du chômage n'est pas uniquement celui du drogué ou du chômeur (qui sont ici plutôt des victimes), mais plutôt le drame de la société.

Or, plusieurs auteurs en psychologie communautaire constatent qu'il y a un hiatus pas toujours facile à franchir entre une théorie des problèmes sociaux et une théorie du changement social. Quelques raisons peuvent expliquer cet état de fait.

Premièrement, bien que la psychologie communautaire insiste sur la nécessité de surmonter les barrières conceptuelles freinant l'élaboration de "paradigmes sociaux » et de solutions au problème d'inéquité sociale, Heller (1989) et Elias (1987) déplorent le fait que les pratiques préventives qui en découlent mettent surtout l'accent sur la psychologie des personnes. L'analyse entrevoit des horizons que l'action n'occupe pas. En d'autres termes, les théories soulèvent des espoirs; le réalisme pragmatique rattrape l'intervenant. C'est ainsi que les objectifs sont, en règle générale, orientés vers le développement de mécanismes d'adaptation individuels au lieu de s'adresser directement à l'élimination des conditions nuisibles qui sont à l'origine des stress vécus individuellement par les jeunes et leur famille (Price et al., 1988). C'est le cas des programmes centrés sur le processus de résolution de problèmes élaborés par D'Zurilla 
et Goldfried (1971 dans Heller, 1990), ainsi que par Spivack et Shure (1974, 1985 dans Heller, 1990). Un consensus de plus en plus clair émerge selon lequel l'entraînement aux compétences sociales $n^{\prime}$ est pas suffisant lorsque parallèlement coexistent des normes culturelles défavorables ou des conditions socio-économiques qui maintiennent le comportement indésirable (Caldwell et al., 1988).

Deuxièmement, force nous est d'admettre les lacunes considérables observées lorsqu'il s'agit de documenter de façon précise la nature des relations entre événements macro-sociaux et problématiques individuelles. Il est en effet difficile de déterminer comment certains contextes environnementaux tels que les politiques économiques, le chômage et l'organisation urbaine influencent la santé mentale et le bien-être psychologique des individus (Dooley et al., 1987 dans Heller, 1990; Kessler et al., 1987 dans Heller, 1990). Or, nous avons besoin de répondre à ce type de question pour établir les plans d'action en matière de prévention. À titre d'exemple, les études épidémiologiques de Brenner (1973) ont révélé qu'en périodes de récession économique et d'augmentation du taux de chômage, on observait de façon concomitante une augmentation des taux de mortalité, $d^{\prime}$ homicide, d'emprisonnement ainsi que d'admission dans les hôpitaux psychiatriques (Bouchard, 1987). Toutefois, il s'est avéré que I'une des hypothèses expliquant le lien entre ces phénomènes aurait trait à l'insuffisance dans le soutien apporté par le réseau pour aider à faire face à ces situations difficiles, les membres du réseau étant eux mêmes affaiblis puisque surchargés et préoccupés par la résolution des problèmes qu'entraînent ces périodes de récession économique (Dooly et al., 1980, 1981 dans Bouchard, 1987). II existerait donc dans ce cas une relation indirecte entre facteurs économiques et troubles émotionnels: cette relation serait médiatisée par le soutien apporté ou non par le réseau, mettant en évidence l'effet tampon ou modérateur du soutien social dans un contexte de stress économique (Dooley et al., 1981; Gore, 1977; Kelly, 1985 dans Bouchard, 1987 ; Tousignant, 1987; Pearlin, 1989). Les objectifs qui découlent d'une telle observation sont doubles et impliquent des choix de société qui dépassent les seules prérogatives de l'intervenant ou du chercheur. Prévient-on la récession (la cause des causes) ou développons-nous des stratégies de soutien lorsque nous devons y faire face (facteur de protection face au stresseur économique) ? Cette réalité fait malheureusement référence à une autre des raisons qui limitent nos capacités d'endiguer les problèmes sociaux chez les jeunes: les logiques politiques et économiques qui conditionnent nos choix de société et qui sont à la base même de l'inéquité.

Une dernière raison rend particulièrement ardues la formulation d'une théorie du changement social et sa mise en application. Les méthodes de recherche et d'évaluation sont peu adaptées pour évaluer les effets 
associés à certains principes identifiés par les tenants de l'approche écologique en psychologie communautaire: les concepts d'interdépendance, de recyclage des ressources, de succession et de développement durable (Mann, 1978). En effet, pour considérer qu'un programme est une réussite, il faudrait pouvoir mesurer les effets sur une longue période de temps et observer les transformations qu'il entraîne dans son sillon, tant dans les milieux proches qu'éloignés (Elias, 1987).

Afin de contrer partiellement la critique selon laquelle les perspectives les plus répandues (et qui s'applique également à Albee) sont trop centrées sur les modifications individuelles et pas suffisamment sur les conditions sociales à l'origine des stresseurs, Elias (1987) propose une nouvelle équation dans laquelle les facteurs socio-environnementaux sont plus étroitement conceptualisés aux dimensions relatives à chaque individu.

fréquence $=\frac{\text { stresseurs }+}{\begin{array}{l}\text { pratiques de } \\ \text { socialisation }\end{array}}$

Ainsi, bien que I'on retrouve les événements stressants dans les équations d'Albee et de Pransky, il est important de mentionner que celle $d^{\prime} E l i a s$ traduit le mieux la réalité des classes sociales moins favorisées. Plus spécifiquement, des études ont démontré que les effets directs et indirects des événements diffèrent notablement selon l'appartenance aux classes sociales. Dans les familles qui ne vivent pas de stress chronique, un événement tel que la naissance d'un enfant peut procurer de la joie et l'adaptation à la transition (naissance) peut se faire plus aisément. Par contre, la venue d'un nouvel enfant dans une famille vivant déjà une situation de stress chronique (ex. : la pauvreté économique) peut augmenter le niveau de stress à moyen ou à long terme (même si à court terme cet événement procure également de la joie). Les recherches de Brown en Angleterre (Brown et Harris, 1978; Lemyre, 1989) et de Belle (1984) ont révélé que la chronicité des conditions de vie des familles pauvres (difficultés liées au logement, à l'emploi, à la situation financière) exacerbe les effets du stress généré par un événement de vie, aussi heureux puisse-t-il être en soi. Dans ces situations, l'apport du soutien social n'est pas suffisant à moyen et à long terme pour modérer les effets des stress chroniques et transitoires, à moins que ce soutien ne soit diversifié et intensif. Mais, à ce moment-ci, deux questions se posent. Les ressources de la famille ou de son environnement social immédiat ne risquent-elles pas de s'épuiser (Dooly et al., 1980, 1981, dans Bouchard, 1987)? Le fait d'être dans un contexte de survie permanente ne risque-t-il pas de miner à la source le réservoir d'énergie disponible, engendrant fatigue, épuisement, sentiment 
dépressif, isolement et leur lot de conséquences sur l'environnement familial (violence familiale) et sur les autres milieux de vie de I'enfant (abandon scolaire, délinquance, etc.)?

Ainsi l'équation doit-elle être appliquée non pas pour chaque individu, mais plutôt pour l'ensemble d'une communauté. Existe-t-il des milieux où une proportion alarmante de familles et de jeunes est dans une situation où les risques environnementaux, familiaux et individuels sont élevés et où les ressources sociales et personnelles ne font pas le poids, taxées qu'elles sont par l'ampleur et l'intensité des tensions dans l'ensemble de la communauté?

\section{Le sous-développement social, la complexité des problèmes sociaux et l'inégalité d'accès à la santé}

Les observations colligées dans le rapport Deux Québec dans un traduisent assez bien l'exercice qu'a réalisé Elias sur le plan théorique. Ainsi, il y est précisé un lien existant entre la présence élevée $d^{\prime}$ 'individus à problèmes multiples et le sous-développement local (Conseil des affaires sociales, 1989). Essentiellement, les mêmes indicateurs définissent les territoires où vivent les personnes défavorisées, en milieu rural comme en milieu urbain (taux de pauvreté, taux de chômage, niveau de scolarité, état de santé, mésadaptation juvénile, handicap socio-pédagogique et déclin démographique (Conseil des affaires sociales, 1989: 68). Les carences qui se manifestent chez plusieurs personnes seraient des manifestations diverses d'un même problème social, des symptômes d'un même phénomène: le sous-développement local. En effet, la fréquence élevée de personnes à problèmes multiples se retrouve dans des lieux géographiques très précis. Par exemple, la répartition géographique du sous-développement social dans la région du Montréal métropolitain est demeurée à peu près statique de 1978 à 1986, mettant davantage en évidence les liens entre conditions socio-économiques et présence de problèmes individuels (Conseil des affaires sociales, 1989).

Selon Côté (1987), deux autres facteurs explicatifs semblent contribuer au sous-développement (surtout dans les régions périphériques des grandes agglomérations urbaines): la perte d'emplois ayant pour cause les fermetures $d^{\prime}$ usines de la ville ou de la région et les transferts fiscaux investis dans les régions économiquement plus intéressantes ${ }^{1}$.

Dans le même ordre d'idées, Paquet (1989) constate que des problèmes individuels comme des troubles de santé se concentrent dans les régions présentant des difficultés socio-économiques; les inégalités sociales en matière de santé sembleraient non seulement se maintenir, mais seraient en hausse croissante ${ }^{2}$. L'écart dans l'espérance de vie serait de 
13 ans entre certains quartiers. Le taux d'incapacité permanente des Québécois âgés de 45 à 64 ans est de 1,8\% chez les cadres et de $40 \%$ chez les travailleurs de l'industrie primaire. D'après une étude menée à Montréal et corroborant les observations de Garbarino aux État-Unis (1978), le taux de mauvais traitement serait 2,8 fois plus élevé dans les secteurs pauvres que dans les secteurs riches (Chamberland, Bouchard et Beaudry, 1986). Cette surreprésentation des problèmes sociaux et de santé dans les classes défavorisées est observée de façon beaucoup trop récurrente pour qu'elle soit uniquement un effet du hasard.

Et pourtant, comment expliquer cette situation malgré la présence de nombreux programmes universels et gratuits d'accès aux soins de santé et la création de programmes d'éducation sanitaire qui font pourtant la fierté d'un pays qui se targue d'être plus avancé que ses voisins sur le plan des politiques sociales? Paquet rétorque à ce sentiment de suffisance que les populations pauvres ont peu accès au pouvoir, à l'avoir et au savoir. Elles sont victimes $d^{\prime} u n$ processus d'exclusion politique, sociale et culturelle des modes de vie "normaux» dans une société (Paquet, 1989: 34). Elles manquent de ressources nécessaires à la pleine participation et aux activités valorisées socialement. Des recherches conduites dans le cadre de la Commission Rochon (Provost, 1988; Lesemann, 1988) en arrivent à la même conclusion. En effet, les jeunes travailleurs (15-19 ans) et les enfants des familles monoparentales sont les plus à risque de subir les effets de l'exclusion sociale étant donné leur situation de pauvreté économique et éducationnelle.

L'une des dimensions contribuant à la dynamique de l'exclusion sociale est d'ordre culturel. Plus particulièrement, l'écart existant entre deux cultures, celle des gens défavorisés économiquement et celle de la classe des professionnels (intervenants en santé et en services sociaux) est trop grand. Les valeurs véhiculées à travers les objectifs d'intervention dans la planification des services de santé et des services sociaux, I'utilisation d'un "langage technique " par les professionnels (savoir) ainsi que la non-reconnaissance de la capacité des gens de définir eux-mêmes leurs besoins et de participer à l'élaboration des solutions dans la résolution de problèmes représentent des facteurs ayant creusé le fossé dans la relation aidant-aidé. Or, cette relation a placé les gens dans une position d'infériorité qui compromet sérieusement la sauvegarde d'une dignité et d'un sentiment de confiance indispensables pour lutter devant une certaine impression diffuse et sournoise $d^{\prime}$ impuissance. Ce ne sont certainement pas des ingrédients favorables au développement de la santé mentale de l'ensemble de la population.

L'analyse de Paquet trouve des appuis dans les études qui mettent le focus sur les facteurs de réussite des projets en prévention. Quelques 
conditions sont préalables à leur succès, notamment la transformation des rôles professionnels (Weissbourg et Kagan, 1989; Guay, 1984), la démocratisation du savoir (Doucet et Favreau, 1991 ; Moreau, 1987), la formation des professionnels en fonction des valeurs, des croyances et des pratiques du milieu (Groupe de travail pour les jeunes, 1991 ; MSSS, 1989) et des attentes de la communauté (Weissbourg et Kagan, 1989) ainsi que l'adoption de stratégies parfois conflictuelles telles que recommandées par l'approche structurelle dans le domaine de l'intervention sociale (Moreau, 1987).

À partir du moment où l'on reconnaît avoir à modifier les relations de pouvoir entre les individus ou la collectivité d'une part et l'environnement économique, politique et culturel d'autre part, et qu'il existe des résistances pour provoquer un tel changement, le recours à des stratégies conflictuelles s'impose. Nous aborderons deux grandes catégories d'approche en intervention qui ont tenté d'améliorer la position des plus démunis sur l'échiquier social. Les moyens expérimentés peuvent, croyons-nous, s'appliquer avantageusement dans le domaine de la prévention des problèmes sociaux dans la mesure où l'approche qui y est privilégiée comprend des solutions où les dimensions personnelles de I'intervention sont conjuguées aux dimensions plus sociales.

\section{DÉVELOPPEMENT D'UNE NOUVELLE VISION LIANT LA RÉSOLUTION DES PROBLÈMES INDIVIDUELS À CELLE DES PROBLÈMES SOCIAUX}

Les situations d'inégalités et d'exclusion sont très présentes dans la chaîne causale provoquant l'apparition des problèmes chez les jeunes. II est donc pertinent de s'interroger sur les pratiques sociales les plus susceptibles $d^{\prime}$ agir sur les déterminants plus éloignés de leurs milieux de vie. Différentes pratiques découlant de traditions théoriques diverses que sont l'empowerment, les pratiques de conscientisation et les mouvements sociaux (Maheu et Descent, 1990) apparaissent liées de très près à tout cet effort collectif de transformation sociale. Cet ensemble de pratiques sociales, parfois innovatrices, occupe une place de choix à l'intérieur du cadre conceptuel en prévention sociale.

\section{L'empowerment et l'organisation communautaire}

L'empowerment est perçu par plusieurs chercheurs comme la capacité que possède l'individu d'agir sur ses différents environnements ou de se percevoir capable de modifier les conditions susceptibles de l'affecter. Swift et Levin (1987) écrivent: 
Empowerment : 1) refers both to the phenomenological development of a certain state of mind (e.g., feeling powerfull, competent, worthy of esteem, etc.) and the modification of structural conditions in order to reallocate power (e.g., modifying the society's opportunity structure) - in other words, empowerment refers both to the subjective experience and the objective reality; and 2) is both a process and a goal (p. 73).

Dans le domaine des pratiques préventives, il peut s'agir d'un objectif $d^{\prime}$ intervention comme tel ou d'une stratégie qui fait appel au processus par lequel une personne gagne du pouvoir sur sa propre vie, développe une connaissance critique des structures de pouvoir dans la société et contribue avec $\mathrm{d}^{\prime}$ autres à transformer les conditions sociales et à réduire les inégalités entre les différents groupes sociaux (Breton, 1992; Cochran, 1986; Rappaport, 1981 ; Swift et Levin, 1987). La dimension objective de la réalité demande donc que la personne puisse reconnaître les obstacles au développement situés hors de son contrôle. Cochran (1986) inclut, parmi ces obstacles, la structure des classes sociales, les différences fondées sur la race et le genre, de même que l'influence du contrôle exercé par la bureaucratisation sur les individus et les groupes sociaux. Traditionnellement, ces obstacles ont plutôt eu tendance à être occultés par les écoles de pensée en psychologie.

Par conséquent, l'empowerment envisagé du point de vue de l'individu et des collectivités vise la modification ou la création de contextes environnementaux (milieux de vie, structures institutionnelles - économiques, valeurs sociales, etc.) favorisant un bien-être individuel et collectif. II conduit par ailleurs à l'acquisition d'une identité personnelle et collective (Mucchielli, 1986). Pour les tenants de cette approche, l'intervention individuelle n'est qu'une étape vers le développement de stratégies $d^{\prime}$ 'action orientées vers des systèmes sociaux plus larges. Les effets souhaités sont à la fois individuels (bien-être psychologique), collectifs (insertion dans des groupes d'entraide) et communautaires (revendication pour l'amélioration des conditions de vie).

Dans la méthode de travail auprès des groupes, on trouve aussi une orientation axée sur I'humanisation des services et sur le développement communautaire. Même si cette méthode a surtout reposé, dans la dernière décennie, sur le modèle de courant central (Papell et Rothman, 1983), on retrouve dans les dernières années des pratiques centrées davantage sur des objectifs qui ont une dimension communautaire. Particulièrement, nous pensons ici au modèle de groupe autogéré (Mullender et Ward, 1991) dans lequel on donne la priorité, même dans des organismes plus traditionnels, à des objectifs de niveau organisationnel et collectif, en tenant pour acquis que les changements individuels découleront du travail sur ces objectifs précités. 
Le fait de considérer la prévention sous l'angle élargi des problèmes sociaux nous amène également à considérer le champ de l'organisation communautaire, les grilles d'analyse dont elle s'inspire et les modèles d'action qu'elle a développés. En effet, bien que l'organisation communautaire n'ait pas utilisé le mot prévention au cours des années 70 , les populations s'organisaient par elles-mêmes afin de répondre à leurs besoins. En fait, des actions ont pris forme lorsque ces populations ont pris conscience des effets négatifs de l'inaction des gouvernements ou de certaines décisions économiques et politiques sur leurs conditions de vie.

Parmi les trois grands courants théoriques qui ont traversé l'organisation communautaire, deux modèles paraissent correspondre aux buts poursuivis en prévention sociale: le développement local et l'action sociale (Rothman, 1968, dans Doucet et Favreau, 1991).

Ces modèles de résolution de problèmes sociaux s'avèrent singulièrement pertinents sous un certain nombre $d^{\prime}$ aspects : leur finalité sur le plan des actions privilégiées, leurs stratégies de changement, les principaux rôles de l'intervenant, leur attitude face à la structure de pouvoir et leur perception de la population concernée.

Le développement local consiste en des luttes locales contre la pauvreté et met l'accent sur les capacités d'auto-développement d'un milieu (ex.: coopérative $d^{\prime}$ habitation, garderie populaire, cuisine collective, etc.). Ainsi, les groupes communautaires peuvent se regrouper en corporation de développement communautaire ou en corporation de développement économique communautaire pour accroître leur pouvoir politique et contribuer activement au développement économique régional.

L'action sociale regroupe les personnes les plus démunies et définit surtout ses actions dans une perspective de défense des droits sociaux. $S^{\prime}$ inspirant du syndicalisme, $c^{\prime}$ est le modèle le plus conflictuel. Ses stratégies ont pour but de développer des rapports de force, des actions politiques pour mener à un changement dans les structures sociales. Les mouvements sociaux, dans leurs stratégies de contestation et de revendication, empruntent à ce modèle $d^{\prime}$ action sociale. Les personnes sont vues comme étant exploitées et opprimées individuellement et collectivement.

En somme,

contrairement à la tradition de l'aide sociale, l'organisation communautaire ne s'intéresse pas aux classes populaires (dans leur milieu de travail ou de vie) parce qu'elles sont faibles (psychologiquement, socialement...), mais pour la force réelle et potentielle qu'elles peuvent représenter [...] Pour être véritablement efficace, l'organisation communautaire doit aussi contribuer à bâtir des organisations dans lesquelles la collectivité locale a le sentiment $d^{\prime}$ 'augmenter son pouvoir, son influence et où les gens considèrent 
être en train de changer l'ordre des choses (Doucet et Favreau, 1991 : 8-10).

En ce sens, ses perspectives coïncident très bien avec la perspective développée par l'approche d'empowerment dans la mesure où l'accent mis sur la capacité $d^{\prime}$ action de l'individu se voit multiplié dans ses effets lorsqu'il est intégré au sein d'une capacité collective.

\section{À PROPOS D'UNE DÉFINITION PRÉLIMINAIRE DE LA PRÉVENTION SOCIALE}

Afin de synthétiser l'ensemble des aspects discutés dans cet article, une réponse aux principales questions posées au début de ce texte permettra de dégager une première esquisse de ce qui pourrait être une définition acceptable de la prévention sociale. $D^{\prime}$ abord, il nous semble plus prudent $\mathrm{d}^{\prime}$ adopter une double perspective pour définir ce sur quoi porteront les efforts en matière de prévention. En premier lieu, l'approche écologique invite à conceptualiser les problèmes individuels comme I'expression d'une difficulté d'adaptation à un environnement en saisissant bien sûr que la demande ou la pression à s'adapter provient souvent des milieux. Toutefois, cette approche insiste aussi sur l'interaction individu-milieu et souligne le caractère actif de l'individu dans son milieu. Néanmoins, le pouvoir qu'a celui-ci de modifier son environnement va précisément dépendre de l'importance des ressources personnelles, sociales, culturelles et économiques dont il dispose. À l'inverse, des lacunes dans ces ressources exposeront la personne à être plus dépendante des forces synergétiques externes qui auront plus tendance à agir unidirectionnellement sur elle. C'est ainsi que l'environnement est souvent perçu comme élément prépondérant du problème, les manifestations individuelles étant l'expression d'un malaise environnemental (Chamberland et Beaudry, 1989). De plus, la perspective écologique (de tendance plus socioculturelle) fournit une analyse des problèmes sociaux plus satisfaisante dans la mesure où les facteurs de risque et de protection examinés sont multiples, interactifs et relatifs; c'est un modèle qui rend compte plus adéquatement de la chaîne causale non linéaire qui intervient dans l'étiologie des problèmes sociaux.

En second lieu, les tenants de l'approche constructiviste soulignent l'importance d'adopter un point de vue critique et non définitif dans nos dénominations et définitions de ce qu'est une problématique sociale. Il nous semble que la pratique du doute raisonnable valorisée par cette approche constitue la voie la plus appropriée pour jauger des réalités sociales très mouvantes où l'évaluation est empreinte de juge- 
ments de valeur qui s'élaborent dans un contexte institutionnel, culturel, géographique, politique et économique bien précis (Mayer et Laforest, 1990).

En outre, les cibles (qui et où) privilégiées sont plutôt les populations en général afin de sauvegarder les principes d'accessibilité et d'universalité des services, lesquels furent déterminants dans le développement des politiques sociales tant au Québec qu'au Canada. De plus, les interventions doivent non pas seulement se diriger vers des groupes de personnes, mais vers les différents milieux proches ou éloignés qui affectent le cours du développement des individus (Garbarino, 1982). Dans le cas où des restrictions dans la disponibilité de ressources humaines ou financières s'imposent, une approche centrée sur les territoires aux prises avec un haut taux de problèmes est privilégiée; ce choix réduit les probabilités que les individus dits à risque soient stigmatisés. II peut toutefois générer un nouvel effet pervers : celui des milieux étiquetés à risque.

Afin de réussir véritablement à atteindre l'objectif formulé en termes de réduction des problèmes sociaux, l'intervention sociale et préventive doit accorder une priorité à l'action proactive (prévention primaire) (quand). De plus, elle doit arrimer son action autour d'objectifs formulés non pas uniquement en fonction de l'évitement de problèmes, mais aussi (et surtout) en fonction de la promotion des outils nécessaires pour que les individus et les collectivités acquièrent et exercent un plus grand contrôle sur leurs conditions de vie et puissent les améliorer.

Conformément au choix théorique énoncé plus haut, soit le recours à des modèles de prévention écologique $d^{\prime}$ inspiration plus socioculturelle, il apparaît dès lors cohérent de privilégier des moyens d'action susceptibles de développer des ressources à la fois chez les individus et dans les communautés, afin que ceux-ci puissent exercer un contrôle efficace sur leur environnement social, politique et économique et soient ainsi avantagés dans leur rapport au pouvoir.

Dans ce contexte, les stratégies d'appropriation (empowerment) et de développement communautaire constituent des moyens pertinents (comment) qui peuvent être avantageusement enrichis par les principes et prémisses énoncés dans l'approche structurelle (Moreau, 1987) et la théorie des mouvements sociaux de Touraine $(1978,1981)$. Toutes deux soulignent l'importance de tenir compte de la dimension conflictuelle dans I'analyse des problèmes sociaux et de l'intervention. Elles mettent en évidence le fait que les problèmes sociaux sont le produit de rapports sociaux conflictuels qui existent entre divers groupes d'intérêts dans une société. Par conséquent, les stratégies mises au point ont pour objectif principal de transformer la direction des rapports de force ( " les levées de boucliers »). 
La prévention sociale pourrait donc s'inscrire dans un mouvement social, dont les efforts concertés entre différents paliers (social - économique - politique - culturel) s'orienteraient vers la résolution durable des principaux problèmes sociaux. La cible ne serait pas uniquement les individus ou les clientèles aux prises avec des risques, mais impliquerait des actions plus globales diversifiées et concertées dans différents contextes : école, quartier, milieu de travail, municipalité, région. Des plans d'action structurants s'avèrent désormais indispensables pour aspirer infléchir l'aggravation de la situation des jeunes et des adultes. Pransky (1991) propose un modèle conceptuel qui intègre de manière cohérente les initiatives à l'intérieur de ce qu'il appelle la pyramide de la prévention primaire. II y décrit un ensemble de stratégies combinant les changements sociaux (la base de la pyramide), la promotion de services de qualité en périnatalité, des programmes de soutien et d'éducation aux parents, des interventions diversifiées en contextes scolaire et de travail, le développement communautaire ainsi qu'une série d'actions de sensibilisation et de marketing social ayant pour véhicule les différentes catégories de médias. En somme, mettre en place une panoplie $d^{\prime}$ 'initiatives susceptibles de créer une force d'impact multipliée par l'effet synergétique des unes et des autres. Bref, pouvoir simultanément réduire les facteurs de risque identifiés au numérateur des équations de Albee (1983), Pransky (1991) et Elias (1987) et augmenter les facteurs de protection situés au dénominateur. Ainsi chaque intervenant devrait percevoir son action dans un plan d'ensemble où la somme est plus grande que ses parties (voir la notion de «Big Picture» dans Pransky, 1991).

Les éléments d'une définition préliminaire de la prévention sociale sont encore à l'état d'ébauche. Ils peuvent toutefois servir à déterminer si des projets spécifiques déjà existants s'avèrent prometteurs et si le qualificatif «social » constitue ou non un abus de langage. Plusieurs facteurs devront ainsi être examinés, tant sur le plan de la conception, de l'implantation que de l'évaluation des effets et des impacts. Premièrement, y-a-t-il une analyse globale des situations problématiques où l'accent est mis sur la synergie des causes? Deuxièmement, a-t-on recours à une variété de stratégies (projets ou actions) ciblant les individus, les milieux de vie et les environnements éloignés? Troisièmement, les objectifs et les moyens sontils démocratiquement déterminés? Quatrièmement, ces projets sont-ils implantés pour plusieurs années afin que les conditions précédemment énoncées puissent avoir l'occasion d'être mises en place? Cinquièmement, l'intervention agit-elle prioritairement vers l'élimination des facteurs prédisposants plutôt que vers des facteurs précurseurs? Sixièmement, intègre-t-elle des dimensions promotionnelles à son plan d'activités? En dernier lieu, l'évaluation d'implantation des effets permet-elle de tirer des 
conclusions pertinentes pour inspirer le développement de projets semblables là où le besoin se fait sentir?

II nous semble que ces conditions doivent être minimalement satisfaites pour réellement entretenir l'espoir de voir réduire de façon durable la prévalence de problèmes sociaux et ainsi prétendre s'engager dans la voie de la prévention sociale.

Au terme de ce texte et en recontextualisant au Québec, il nous semble que la conjonction de plusieurs éléments, comme une plus grande conceptualisation de la prévention, la recherche en ce domaine, la montée d'initiatives en prévention ainsi que les perspectives préventives pour les services, ainsi que le souhaitent de nombreux groupes de travail, pourra marquer une évolution intéressante dans le courant de la prochaine décennie.

\section{Notes}

1. Les économistes Dooly et Catalano (1987) ont traité la question des stratégies de protection contre le déclin économique des régions. Pour eux, cette priorité est essentielle en prévention. Ils préconisent une évaluation de l'impact et une analyse des coûts / bénéfices des décisions économiques, particulièrement sur la santé mentale. Ces études pourraient mettre en évidence, pour le bénéfice des électeurs, le système des valeurs des institutions et des législateurs. Ils proposent à titre de mesures préventives la taxation des régions en expansion pour aider celles en déclin, de même que des mesures obligeant les entreprises à assumer des responsabilités sociales (entre autres le coût des problèmes de santé mentale lié à la mobilité démographique) (dans Jason et al., 1987).

2. Le rapport de la commission Rochon sur les services de santé et les services sociaux a également souligné que si l'espérance de vie a augmenté, l'espérance de vie en bonne santé de certaines populations n'a certes pas fait de même.

\section{Références bibliographiques}

ALBEE, G.W. (1982). "The politics of nature and nurture», American Journal of Community Psychology, vol. 10, $\mathrm{n}^{\circ} 1:$ 4-36.

ALBEE, G.W. (1983). "Psychopathology, prevention and the just society", Journal of Primary Prevention, vol. 4, $\mathrm{n}^{\circ} 2: 5-40$.

ALBEE, G.W. (1986). "Toward a just society: lessons from observations on the primary prevention of psychopathology ", American Psychologist, vol. 41, $\mathrm{n}^{\circ}$ 8: 891-898.

AlbeE, G.W. (1980). Social Science and Social Change. The Primary Prevention of Disturbance in Youth. Burlington, VT: University of Vermont. 
BELLE, D. (1984). Lives in Stress. Women and Depression. Beverly Hills : Sage Publications.

BLOOM, B.L. (1979). «Prevention of mental disorders : recent advances in theory and practice», Community Mental Health Journal, vol. 15 : 179-191.

BLOOM, B.L. (1981). "The logic and urgency of primary prevention », Hosp. Community Psychiatry, $32: 839-843$.

Bloom, M. (1980). Primary Prevention: The Possible Science. Englewood Cliffs (N.J.) : Prentice-Hall.

BOUCHARD, C. (1987). Revenus, emplois, réseaux sociaux et voisinages: leur influence sur les relations intra-familiales. LAREHS, 19 pages.

BOUCHARD, C. (1987). Vous avez dit l'approche écolosystémoglobal-holisticofamilio-communautariste? Comment s'y perdre. Conférence présentée au colloque régional sur les compétences parentales, DSC Saint-Luc, Montréal, mai, 12 p.

BOWER, E.M. (1990). "La promotion du fonctionnement humain : un guide pour l'idéaliste pragmatique ", dans Prévention psychosociale pour l'enfance et I'adolescence, sous la dir. de J.-F. Saucier et L. Houde. Montréal: Presses de I'Université de Montréal, p. 315-368.

BRENNER, M.H. (1973). Mental IIIness and the Economy. Cambridge: Harvard University Press.

BRETON, M. (1992). Empowerment et groupes en service social, allocution dans le cadre du symposium sur la prévention sociale et ses applications. Montréal, mai.

BRONFENBRENNER, U. (sous presse). "The process-person-context model in developmental research : principles, applications and implications », dans: Enfance et famille: contextes de développement, sous la dir. de R. Tessier, C. Bouchard et G. M. Tarabulsy. Québec: Presses de I'Université Laval.

BROWN, G. W. et T. HARRIS (1978). Social Origin of Depression, Londres: Tavistock.

CALDWELL, R.A., G.A. BOGAT et W.S. DAVIDSON II (1988). "The assessment of child abuse potential and the prevention of child abuse and neglect: a policy analysis ", American Journal of Community Psychology, vol. 16, $\mathrm{n}^{\circ} 5$ : 609-624.

CAtAlino, R. (1979). Health, Behavior and the Community: An Ecological Perspective. New York: Pergamon Press.

CHAMBERLAND, C. (1992). Réflexions d'inspiration galiléenne: implications vis-à-vis la prévention (sous presse).

CHAMBERLAND, C. et C. BOUCHARD (1990). "Communautés à risques faibles et élevés de mauvais traitements : points de vue d'informateurs-clés », Service social, vol. 39, n 2 : 76-101.

Chamberland, C., C. BOUCHARD et J. BEAUdRY (1986). «Les mauvais traitements envers les enfants : réalités canadienne et américaine », Revue canadienne des sciences du comportement, vol. 18: 391-412.

CHAMBERLIN, R.W. (1984). "Strategies for disease and health promotion in maternal and child health: the "ecologic" versus "high risk" approach", Journal of Public Health Policy, vol. 5, $\mathrm{n}^{\circ} 2$ : 185-197. 
CHIN, R. et K. BENNE (1985). "General strategies for effecting changes in human systems ", dans W. Bennis, K. Benne et R. Chin, The Planning of Change. New York: Holt, Rinehart et Winston, p. 32-59.

COCHRAN, M. (1986). "The parental empowerment process: Building on family strengths », dans J. Harris (ed.), Child Psychology in Action : Linking Research and Practice.

COMITÉ BRUNET (1987). Rapport du comité de réflexion et d'analyse des services dispensés par les CLSC. Québec: Gouvernement du Québec, ministère de la Santé et des Services sociaux.

CONSEIL DES AFFAIRES SOCIALES (1989). Deux Québec dans un : rapport sur le développement social et démographique. Chicoutimi : Gaëtan Morin, $124 \mathrm{p}$.

CÔTÉ, C. (1987). "Le développement de la pauvreté ", dans Les nouvelles pauvretés, sous la dir. de M. Gauthier. Montréal: IQRC.

DOUCET, L. et L. FAVREAU (1991). Théorie et pratiques en organisation communautaire. Sainte-Foy: Presses de l'Université du Québec, 464 p.

ELIAS, M.J. (1987). "Establishing enduring prevention programs: advancing the legacy of Swampscott ", American Journal of Community Psychology, vol. 15, $\mathrm{n}^{\circ} 5$ : 539-553.

FORTIN, A. (1992). "Le mauvais traitement psychologique: une réalité encore mal connue ", PRISME, vol. 3, no $1:$ p. 88-100.

GARBARINO, J. (1983). "Social support networks: Rx for the helping professionals ", dans Social Support Networks: Informal Helping in the Human Services, sous la dir. de J. K. Whittaker et J. Garbarino. New York: Aldine Publishing Company.

GARbarino, J. et A.C. CROUTES (1978). "Defining the community context of parent-child relations", Child Development, vol. 49: 604-616.

GOFFMAN, E. (1975). Stigmate, les usages sociaux des handicaps. Paris: Minuit.

GOUVERNEMENT DU QUÉBEC (1985). Prévenir, traiter et réadapter efficacement, vol. 2. Avis du Comité de la santé mentale du Québec. Québec: Publications du Québec.

GOUVERNEMENT DU QUÉBEC (1990). Une réforme axée sur le citoyen. Québec: Ministère de la Santé et des Services sociaux.

GOUVERNEMENT DU QUÉBEC (1991). Un Québec fou de ses enfants. Rapport du groupe de travail pour les jeunes. Québec: Ministère de la Santé et des Services sociaux.

GOUVERNEMENT DU QUÉBEC (1992). La politique de la santé et du bien-être. Québec: Ministère de la Santé et des Services sociaux.

GUAY, J. (1984). L'intervenant professionnel face à l'aide naturelle. Chicoutimi : Gaëtan Morin éd.

HELLER, K. (1989). "The return to community ", American Journal of Community Psychology, vol. 17, $\mathrm{n}^{\circ} 1: 1-15$.

HELLER, K. (1990). "Social and community intervention ", Annual Review of Psychology, vol. 41 : 141-168.

JASON, L.A., R.E. HeSS, R.D. FELNER et J.N. MORITSUGu (1987). Prevention: Toward a Multidisciplinary Approach. New York: Haworth. 
KELLY, J.C. (1968). "Toward an ecological conception of preventive interventions ", dansResearch Contributions from Psychology to Community Mental Health, sous la dir. de J. W. Carter. New York: Behavioral Publications.

KLEIN, D.C. (1987). "The context and times at Swampscott : my story ", American Journal of Community Psychology, vol. $15 \mathrm{n}^{\circ} 5:$ 531-553.

LAFORTUNE, D. et M.C. KIELY (1989). "Prévention primaire des psychopathologies: appellation contrôlée", Santé mentale au Québec, vol. 14, n 1 : 54-68.

Laurendeau, M.-C., R. Perreault et L. BlanChet, (1990). "La promotion de la santé mentale ", Santé et Société, Cahier n ${ }^{\circ} 5$.

LEMYRE, L. (1989). "Stresseurs et santé mentale : analyse contextuelle de la pauvreté ", Santé mentale au Québec, vol. 14, n² 2: 120-127.

LESEMANN, F. (1987). Les nouvelles pauvretés: I'environnement économique et les services sociaux, Commission d'enquête sur les services de santé et les services sociaux, annexe 4, Gouv. du Québec.

LoW, K. (1992). Prevention - Correction Time Line, allocution dans le cadre du symposium sur la prévention sociale et ses applications. Montréal, mai.

LoW, K. (1979). "La prévention ", dans Connaissances de base en matière de drogue, Manuel n ${ }^{\circ}$ 5. Ottawa : Santé et Bien-être social.

MAHEU, L. et D. DESCENT (1990). "Les mouvements sociaux : un terrain mouvant ", Nouvelles pratiques sociales, vol. 3, $n^{\circ} 1: 41-52$.

MANN, P. A. (1978). Community Psychology: Concepts and Applications. New York: The Free Press, $339 \mathrm{p}$.

MASSÉ, R. (1992). "Construction sociale et culturelle de la maltraitance », PRISME, vol. 3, $\mathrm{n}^{\circ} 1: 12-15$.

MAYER, R. et M. LAFOREST (1990). "Problème social : le concept et les principales écoles théoriques ", Service social, vol. 39, n $2: 1343$.

MOREAU, M. (1987). " L'approche structurelle en travail social: implications pratiques d'une approche intégrée conflictuelle », Service social, vol. 36, $n^{\text {os }} 2-3: 27-47$.

MUCCHIELLI, A. (1986). L'identité. Paris: PUF, Que sais-je?, 127 p.

MULLENDER, A. ET D. WARD (1991). Self-directed Groupwork - Users Take Action for Empowerment. Londres: Whiting and Birch, $194 \mathrm{p}$.

PAPELL, C. et B. ROTHMAN (1983). "Le modèle du courant central du service social des groupes en parallèle avec la psychothérapie et l'approche de groupe structuré», Service social, vol. 32, nos 1-2:11-31.

PAQUET, G. (1989). Santé et inégalités sociales: Un problème de distance culturelle. Montréal: IQRC, $131 \mathrm{p}$.

PEARLIN, L.I. (1989). "The sociological study of stress", Journal of Health and Social Behavior, vol. $30:$ 241-256.

PRANSKY, J. (1991). Prevention: The Critical Need. Burrell Fox Foundation \& Paradigmpers.

PRICE, H.R., E.L. COWEN, P.R. LORION et J. RAMOS-MCKAY (1989). "The search for effective prevention programs: what we learned along the way", American Journal of Orthopsychiatry, vol. 59, $\mathrm{n}^{\circ} 1: 49-58$. 
Provost, M. (1987). Les nouveaux phénomènes sociaux : la catégorie sociale jeunesse. Commission d'enquête sur les services de santé et les services sociaux, annexe 5, Gouv. du Québec.

RAPPAPORT, J. (1981). "In praise of paradox: A social policy of empowerment over prevention ", American Journal of Community Psychology, vol. 9 : $1-25$.

ROSENTHAL, R. et L.F. JACOBSON (1968). "Teacher expectations for the disadvantaged ", Scientific American, 4 : 19-23.

SCHNEIDER, J.W. (1985). "Social problems theory. The constructionist view", Annual Review of Sociology, $11: 209-229$.

SWIFT, C. et G. LEVIN (1987). "Empowerment: An emerging mental health technology ", Journal of Primary Prevention, vol. 8, $\mathrm{n}^{\text {os }} 1-2: 71-94$.

TOURAINE, A. (1978). La voix et le regard. Paris: Seuil.

TOURAINE, A. (1981). Le pays contre l'État. Paris: Seuil.

TOUSIGNANT, M. (1987). Utilisation des réseaux sociaux dans les interventions. État de la question et propositions d'action. Commission d'enquête sur les services de santé et les services sociaux, annexe 9, Gouv. du Québec.

VINCENT, T.A. et E.J. TRICKETT (1983). «Preventive intervention and the human context : ecological approaches to environmental assessment and change ", dans Preventive Psychology: Theory, Research and Practice, sous la dir. de R.D. Felner. New York: Pergamon Press.

WEISSBOURG, B. et L. KAGAN (1989). «Family support programs: catalysts for change ", American Journal of Orthopsychiatry, vol. 59, n $1: 20-31$. 